\title{
Evaluation of a developmental screening system for use by child health nurses
}

\author{
B S L EU
}

Special Services Unit, Child, Adolescent and Family Health Service, South Australian Health Commission, Adelaide, South Australia

SUMMARY An adapted version of the Woodside system for screening general development of the child is being used by child health nurses as part of their routine duties. In the present paper the system is validated in terms of standardisation, reliability, and validity. The system was standardised on 444 children aged $0-4$, the assessments being carried out by a fully trained child health nurse. The inter-tester reliability of the system was found to be at least $95 \%$. The validation sample consisted of all 27 'abnormal' children, all 20 'doubtful' children, and 77 'normal' children, and a highly significant correlation was obtained between the Woodside subsection scores and the relevant Griffiths' scales subsection scores. The findings support initial impressions that the adapted Woodside system is accurate. It is recommended for use by child health nurses as part of their developmental surveillance.

The basic premise underlying paediatric developmental screening is that the earlier a dysfunction or defect is identified, the better will be the final outcome for mother or child, or both. ${ }^{1-5}$ Early diagnosis is certainly important with hearing and visual dysfunction, as a delay in beginning appropriate intervention beyond the known 'critical' or 'sensitive' periods may result in irreversible damage or distortion. ${ }^{6-8}$ The benefits in screening for general development are not so obvious, as similar critical periods do not seem to exist. Even so, early intervention will, in many cases, alleviate or improve a developmental dysfunction, while failure to intervene may compound the underlying problem. ${ }^{9}$

If screening for general development can be justified consideration should be given to the suitability of available screening instruments and to the personnel who will apply them. Until recently, developmental screening in Australia was left to the family doctor, who used his experience to make an unstructured assessment of a child. Today, the art and science of developmental screening has become more sophisticated, and its implementation has, in some places, been transferred from qualified medical practitioners to suitably trained, though lesser qualified, personnel. To improve reliability, screening instruments of a more formal nature and with predetermined referral criteria have been devised. One such screening instrument is the Woodside system, ${ }^{10}$ which was devised ten years ago in Woodside, a suburb of Glasgow, Scotland. A version of the Woodside system, adapted for Australian conditions, was introduced seven years ago at the Mothers and Babies' Health Association* for use by child health nurses who were already involved in preventive care of children aged $0-5$. The adapted Woodside system was found to be short and easily administered and could readily be incorporated into the routine duties of the nurses.

It remained, however, for the system to be standardised and validated for Australian children of different ages. The present study (begun in May 1980 ) evaluates the adapted Woodside system as it is used by child health nurses.

\section{The Woodside system}

The Woodside system uses four charts to provide a visual summary of the developmental progress of a child. The charts, covering the areas of 'Social', 'Hearing and language', 'Vision and fine motor', and 'Gross motor' development, are shown in Figure 1(a)-(d). They have been adapted for Australian conditions, mainly in the gross motor area.

*Now part of the Child, Adolescent and Family Health Services, South Australian Health Commission. 
(a)

\section{SOCIAL}

1. Able to dress - except laces and back buttons $(H)$.

2. Dry at night $(\mathrm{H})$.

3. Washes hands $(\mathrm{H})$.

4. Pulls pants up and down $(H)$.

5. Drinks and replaces cup $(H)$.

6. Knows parts of the body (4)

7 Drinks from cup without spilling $(H)$.

8. Indicates toilet needs $(\mathrm{H})$.

9. Puts cubes into box after being shown.

10. Finds toy under cup.

11. Rings bell.

12. Chews and swallows biscuit (observed). Copes with solid food.

13. Puts objects into mouth (cubes).

14. Reaches for and shakes rattle.

15. Responds to friendly face.

16. Enjoys being handled by mother $(H)$.

17. Smiles when spoken to

18. Some vocal sounds $(H)$.

(H) - History of achievement sufficient.

\section{Normal progress}

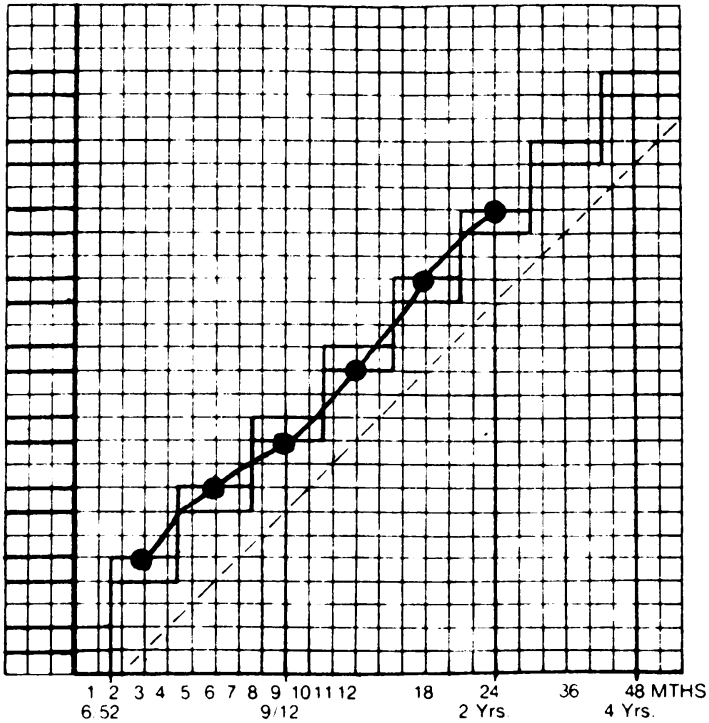

Doubtful progress

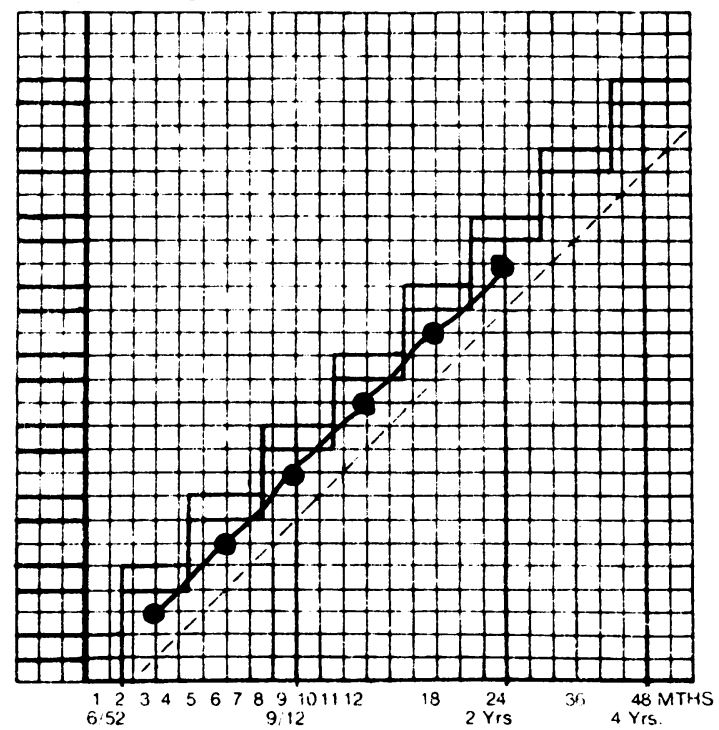

\section{HEARING \& LANGUAGE}

1. 2 or more pronouns in conversation

2. Grammatical speech articulated correctly

3. Says first name.

4. Knows own sex.

5. Simple sentences $(H)$.

6. Plays with miniature cup and saucer.

7. Points to parts of body.

8. Says five or more words $(H)$.

9. Obeys simple commands, e.g. clap hands.

10. Says less than 5 words including "Mama" "Dada" "Baba" (if related to a person)

11. "Mamma" "Dadda" "Baba".

12. Hearing tesis above ear level.

13. Unintelligible babble.

14. Hearing tests at ear level.

15. Turns eyes to sound.

16. Looks round meaningfully when spoken to.

17. Stills to bell.

18. Stills to mother's voice.

$(\mathrm{H})$ - History of achievement sufficient. 
(c)

\section{VISION \& FINE MOTOR}

1. Picks up and replaces very small objects, e.g pins with each eye covered separately.

2. Copies a square.

3. Copies a circle.

4. Builds a bridge of three bricks when shown.

5. Makes a vertical line when shown.

6. Makes a tower of six bricks when shown.

7. Makes a scribble on paper.

8. Makes a tower of three bricks when shown.

9. Pincer grasp using a small object e.g. Smartie.

10. Bangs bricks together when shown

11. Side of finger grasp using a smali object e g Smartie

12. Matches cubes.

13. Picks up cube from table or hand.

14. Transfers cube from one hand to another

15. Holds a pencil briefly.

16. Follows a moving person with eyes.

17 Follows a moving face with eyes

(d)

\section{GROSS MOTOR}

1. Stands on one leg 3-5 seconds.

2. Hops.

3. Stands on leg momentarily.

4. Walks on tiptoe $(\mathrm{H})$.

5. Runs on whole of foot.

6. Kicks ball.

7. Picks object from floor without overbalancing.

8. Kneels without support $(H)$.

9. Pulls to standing on furniture.

10. 'Cruises' round furniture.

11. Sits steadily on floor without support for few mins. $(H)$

12. Stands holding on to furniture.

13. Sits against wall or hand-no lateral support $-2 / 3$ secs

14. Hold round waist, lower abruptly exclude scissoring.

15. Pull from lying. Little or no head lag.

16. Ventral suspension. Holds head above plane of body.

17. Ventral suspension. Head in plane of body.

(H) - History of achievement sufficient.
Abnormal progress

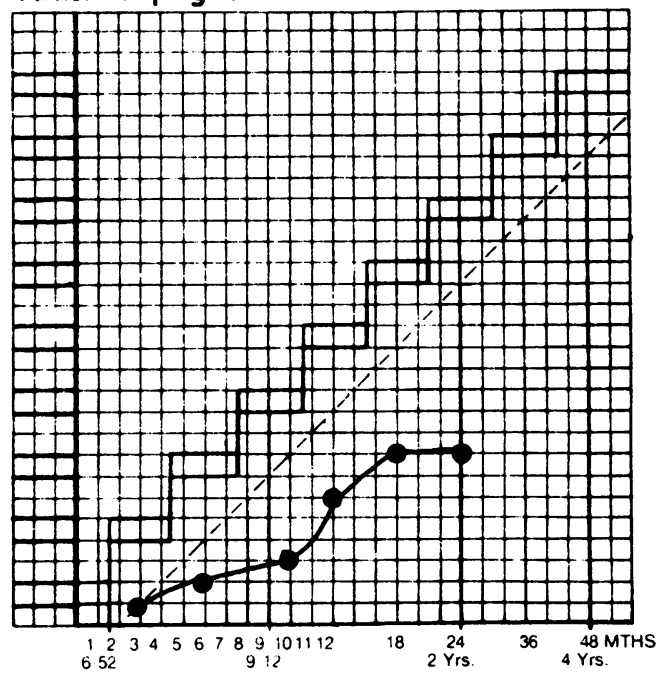

'Fall-off' progress

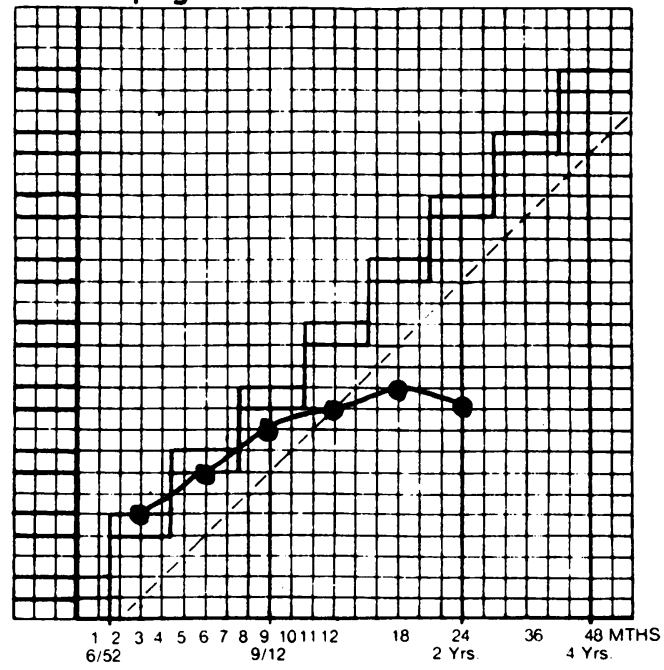

Fig. 1 Charts used in Woodside system to provide a visual summary of the developmental progress of a child: (a) Social; (b) Hearing and language; (c) Vision and fine motor; and (d) Gross motor. 
On the horizontal axis of each chart is shown the age of the child, with a range from 6 weeks to 4 years. The vertical axis contains a variety of tests set out in pairs with the pairs corresponding to appropriate ages and forming a step pattern across the chart. In each of the four charts a child's developmental ability is plotted against the age of the child. In practice there is a minimum total of eight test items to be applied for any individual child-that is, two test items for each of the four developmental areas.

With, for example, a 9 month old child being assessed using the chart on 'Social' development, a mark is made on the upper level of the step if the child succeeds in both test 11 and in test 12 , and at the lower level of the step if he or she succeeds in only one of them (in Figure 2, these two marks are represented by $\mathrm{O}$ and $o$, respectively). If tests 11 and 12 are both failed, the child is assessed on tests
13 and 14, which are normally accomplished by a 6 month old child. Success at one or both of tests 13 and 14 is scored at the appropriate level (X or $\mathrm{x}$ in Figure 2). Tests 15 and 16 are applied if a child fails at tests 13 and 14 (scored V or v in Figure 2). For a bright child tests 9 and 10 might be attempted, which are normally accomplished by a 12 month old (scored $\mathrm{Y}$ or $\mathrm{y}$ in Figure 2). This last procedure is not recommended routinely as the object is to identify delay rather than bright children.

The assessments recorded on the individual charts are interpreted as follows: if the marks on the chart lie on or above the step the child's development in the particular area is suspected to be normal; if the marks lie between the step and the dotted, or 'threshold' line, development is considered to be doubtful; and if the marks lie on or below the dotted line, development is suspected to be abnormal/ delayed.

ADAPTED FROM THE WOODSIDE SYSTEM

NAME: $\quad$ D.O.B.

Interest in surroundings/people.

Activity, appropriate for age.

SOCIAL

1. Able to dress - except laces and back buttons $(H)$.
2. Dry at night $(H)$.
3. Washes hands (H).
4. Pulls pants up and down (H).
5. Drinks and replaces cup (H).
6. Dry by day (H).
7. Drinks from cup without spilling (H).
8. Indicates toilet needs (H).
9. Puts cubes into box after being shown.
10. Finds toy under cup.
11. Apprehensive of strangers.
12. Chews and swallows biscuit (observed).
Copes with solid food (H).
13. Puts objects into mouth (cubes).
14. Reaches for and shakes rattle.
15. Responds to friendly face.
16. Enjoys being handled by mother (H).
17. Smiles when spoken to.
18. Some vocal sounds (H).

$(H)=$ History of achievement sufficient.
Observation: Normal / Doubtful / Abnormal.

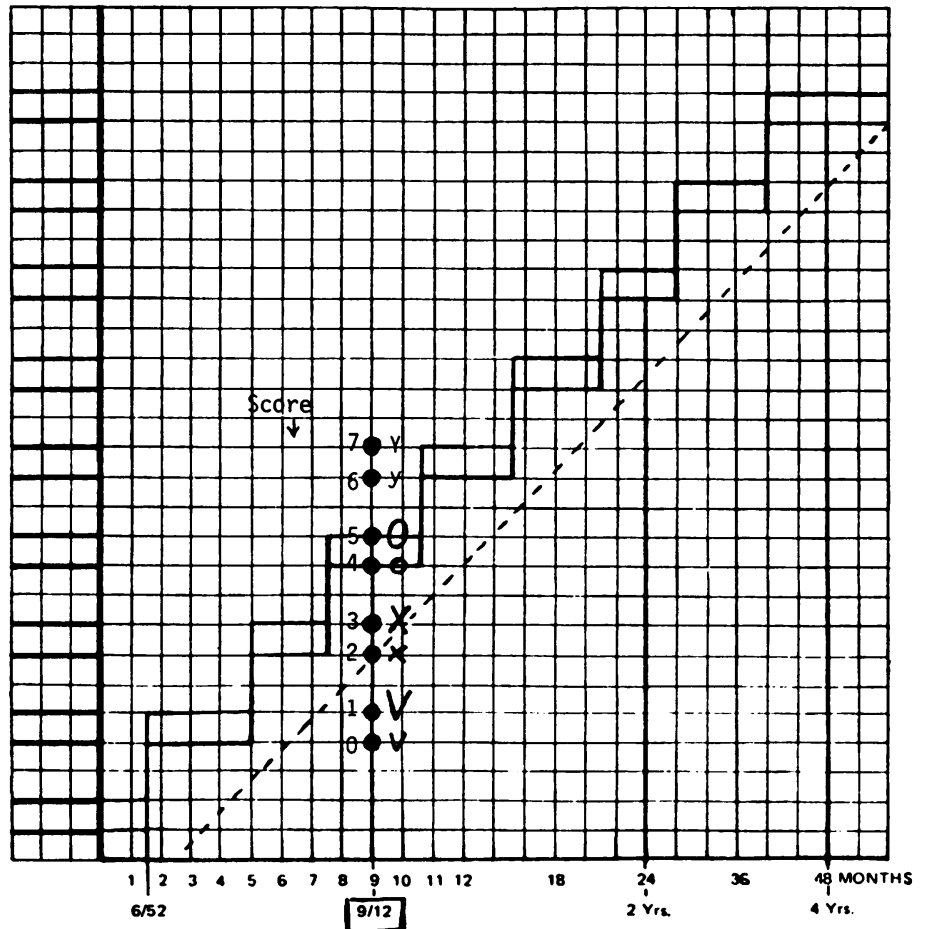

Fig. 2 Example of a 9 month old child assessed for social development (see text for explanation). 
When the assessment plot lies on or below the dotted line in the first year of life the child's development is delayed by roughly three months; in the second year it is delayed by six months; and in the third and fourth years it is delayed by 12 months. For purposes of computer analysis in the present study, a score was allocated to the levels of attainment (in Figure 2)-that is, $Y=7, y=6, O=5$, $\mathrm{o}=4, \mathrm{X}=3, \mathrm{x}=2, \mathrm{~V}=1$, and $\mathrm{v}=0$.

Criteria which are used to decide whether a particular child should be further followed up or referred for appropriate diagnostic assessment are:

(1) If a total of two or more doubtful marks are recorded from the four areas of assessment the child is referred for a full diagnostic assessment.

(2) In the event of one doubtful mark being recorded in the area of 'Gross motor' development, the child is reassessed in two months' time.

(3) If one or more abnormal/delayed marks are recorded the child is referred for a full diagnostic assessment.

(4) If there is a 'fall off' in development from normal at one assessment to doubtful or abnormal/ delayed at a subsequent assessment the child is also referred for a full diagnostic assessment.

The system is most helpful for regular, routine screening as it can be seen at a glance how a particular child is developing. Figure 1(a) shows normal progress, Figure 1(b) doubtful progress, Figure 1(c) abnormal/delayed progress, and Figure 1(d) a gradual fall off in progress.

\section{Sources and methods}

Subjects. The total number of children screened on the adapted Woodside system was 444 , of whom 224 were boys and 220 were girls. Their ages ranged from 6 weeks to 4 years.

To obtain a normal distribution of children, the clinics from which they were drawn were situated in a range of high, medium, and low socioeconomic areas of the suburbs of Adelaide. Socioeconomic state of individual children was designated according to the employment, educational level, and housing condition of the parents of the children. ${ }^{11}$ Altogether, 139 children ( 72 boys and 67 girls) were categorised as being of high socioeconomic state; 184 children ( 87 boys and 97 girls) as being of medium socioeconomic state; and 121 (65 boys and 56 girls) as being of low socioeconomic state.

Also studied were 17 confirmed abnormal children from a spastic centre, who were not included in the normal distribution group. There were nine boys and eight girls, and their age range was from 1 year 10 months to 4 years 4 months.

There were 52 children from ethnic groups, mainly Italian and Greek, which is a representative sample of the Adelaide metropolitan population. Of the 52 children $(12 \%$ of the total of 444$), 11$ were of high socioeconomic state ( $8 \%$ of 139$), 18$ of medium socioeconomic state $(10 \%$ of 184$)$, and 23 of low socioeconomic state $(19 \%$ of 121$)$.

\section{Methods}

A child health nurse screened 461 children (the 444 children in the sample and the 17 known abnormals) using the adapted version of the Woodside system.* Training of the nurse in implementing the assessment was considered to be complete when there was a $95 \%$ agreement with BSLE in the scoring of 10 consecutive children. Details of the application of the Woodside system is described above.

In each of the four areas of development a child was given a score from 0-7 (as described above) on the adapted Woodside system. Scores of 2 or less in any one area or 3 in two or more areas were classified as 'suspected abnormal'; a score of 3 in one area was classified as 'suspected doubtful'; and a score of 4 or more in all areas was classified as 'suspected normal'.

The reliability of the adapted Woodside system was assessed, firstly, with two different testers assessing an individual child on separate occasions yet within a time interval of two weeks ( 50 children with an age range of 6 weeks to 4 years were assessed likewise), and, secondly, with the same tester assessing an individual child on two occasions one week apart (30 children).

A diagnostic assessment, using the Griffiths scales criterion test, ${ }^{12}{ }^{13}$ was performed on all children deemed 'suspected doubtful' or 'suspected abnormal' by the Woodside screening assessment, and on at least $10 \%$ of the total number deemed 'suspected normal'. The diagnostic assessments were performed by two psychologists, a paediatrician, and BSLE, and all assessments were made within two weeks of the nurse's screening assessment with the Woodside system.

Vision and hearing were screened by a child health nurse using the Stycar method. The referral criteria for this screening has been previously described. ${ }^{14}$ Height, weight, and head circumference were measured on all children, and a physical medical examination was performed on 426 children $(96 \%)$.

\section{Results}

Table 1 shows the normative data for the four areas *Full details of the adapted Woodside system can be obtained from BSLE. 
Table 1 Woodside normative data in terms of means (SD) according to socioeconomic groups

\begin{tabular}{lllll}
\hline $\begin{array}{l}\text { Socioeconomic } \\
\text { group }\end{array}$ & Social & $\begin{array}{l}\text { Hearing } \\
\text { and } \\
\text { language }\end{array}$ & $\begin{array}{l}\text { Vision } \\
\text { and fine } \\
\text { motor }\end{array}$ & $\begin{array}{l}\text { Gross } \\
\text { motor }\end{array}$ \\
\hline High $(\mathrm{n}=139)$ & $4 \cdot 6(0 \cdot 6)$ & $4 \cdot 6(0 \cdot 8)$ & $4 \cdot 7(0 \cdot 7)$ & $4 \cdot 7(0 \cdot 9)$ \\
Medium $(\mathrm{n}=184)$ & $4 \cdot 7(0 \cdot 8)$ & $4 \cdot 8(0 \cdot 9)$ & $4 \cdot 7(0.9)$ & $5 \cdot 1(1 \cdot())$ \\
Low $(\mathrm{n}=121)$ & $4 \cdot 7(1 \cdot 1)$ & $4 \cdot 4(1 \cdot 1)$ & $4 \cdot 4(1 \cdot 1)$ & $4 \cdot 8(1 \cdot 1)$ \\
\hline
\end{tabular}

of the adapted Woodside ('Social', 'Hearing and language', 'Vision and fine motor', and 'Gross motor' development) obtained in Adelaide children of different socioeconomic groups.

The reliability of the adapted Woodside system calculated as the percentage agreement in the scores obtained on individual children by the same tester performing the test on two occasions one week apart, was $95 \cdot 5 \%$. The percentage agreement of two different testers making their assessments within two weeks of each other was $95.0 \%$.

Tables 2 and 3 and Figure 3 show the validation studies of the adapted Woodside system, expressed in terms of correlation coefficients, sensitivity (copositivity) and specificity (co-negativity), overreferrals, under-referrals, and regression lines. A total of 124 children, comprising the 27 children assessed as 'suspected abnormal' on the Woodside system, the 20 assessed as 'suspected doubtful', and

Table 2 Correlation between Woodside (screening) system and corresponding Griffith' (criterion) test in the four areas of development in 124 children

\begin{tabular}{ll}
\hline Area of development & Correlation ' $r$ \\
\hline Social & $0 \cdot 46^{*}$ \\
Hearing and speech & $0 \cdot 69^{*}$ \\
Vision and fine motor & $0 \cdot 65^{*}$ \\
Gross motor & $0 \cdot 76^{*}$ \\
\hline Total & $0 \cdot 73^{* *}$ \\
\hline
\end{tabular}

${ }^{*} \mathrm{p}<\left(0 \cdot()(0) 1:{ }^{* *} \mathrm{p}<0 .(1)\right.$.

Table 3 A $2 \times 2$ contingency table showing the number of abnormal scores on the Woodside system against the relevant corresponding Griffiths' score

\begin{tabular}{llll}
\hline Woodside & (iriffiths & Total \\
\cline { 2 - 3 } & Abnormal & Normal & \\
\hline Abnormal & 22 & 5 & 27 \\
Normal & 5 & 72 & 77 \\
\hline Total & 27 & 77 & $104^{*}$ \\
\hline
\end{tabular}

${ }^{*}$ Excluding 20 doubtful cases.

Co-positivity (sensitivity) $22 / 27=01.81$. Co-negativity: (specificity): $72 /$ $77=0.94$.

Over-referrals $=5 / 124=4 \%$. or $5 / 1104=4.8 \%$ (excluding the doubtful cases).

Under-referrals $=5 / 124=4 \%$, or $5 / 104=4 \cdot 8 \%$ (excluding the doubtful cases).

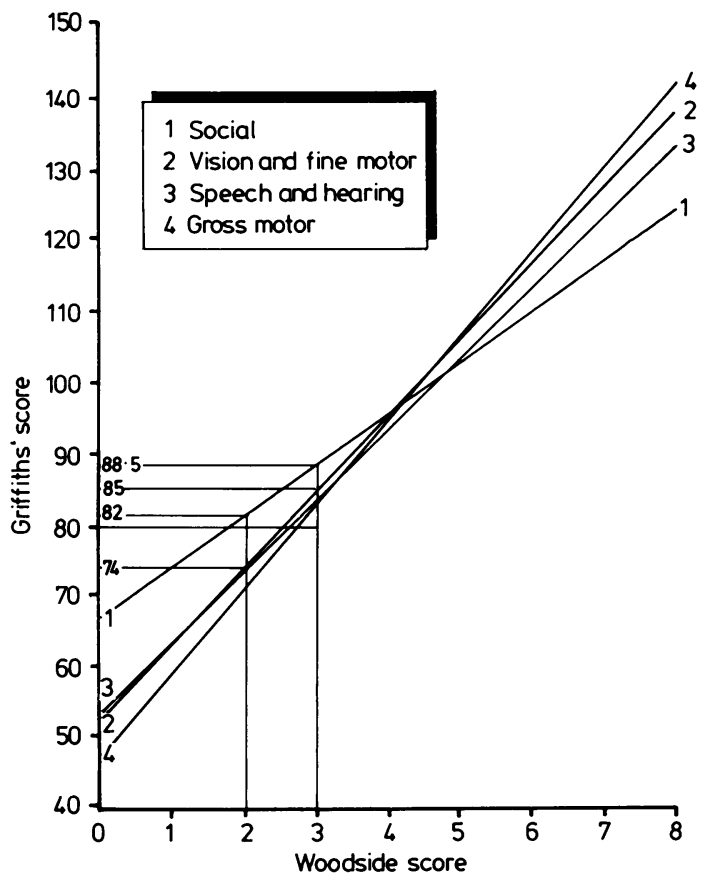

Fig. 3 Regression lines for Woodside scores of social, vision and fine motor, speech and hearing, and gross motor developmental progress plotted against corresponding Griffiths' scores.

77 assessed as 'suspected normal', were retested using the Griffiths' criterion test.

Figure 3 also shows the regression lines for the four areas of the Woodside system plotted against the Griffiths' scores. The four lines represent 'Social', 'Hearing and language', 'Vision and fine motor', and 'Gross motor' development. When considering any one of the four areas of development, a Woodside score of 2 had a corresponding Griffiths' scores (criterion test) of 74 or less in three of the four areas, except for the area of 'Social' development, where the relevant Griffiths' score was 82 . It is reasonable, therefore, to suggest that a Woodside score of 2 would be an acceptable cut off point, as the corresponding Griffiths' score of 74 is just over 2 SD from the mean. Thus a Woodside score of 2 is categorised as 'abnormal'. (The Griffiths' test has a mean score of 100 and a SD of 12).

For a Woodside score of 3 the corresponding Griffiths' score was 85 or less in three of the four areas, except for the area of 'Social' development again, where the Griffiths' score was 88.5. A Woodside score of 3 corresponds with a Griffiths' 
Table 4 Classification of children identified from the Woodside screen according to socioeconomic group $(n=444)$

\begin{tabular}{lllr}
\hline $\begin{array}{l}\text { Socioeconomic } \\
\text { group }\end{array}$ & 'Normal' & $\begin{array}{l}\text { 'Doubtful and } \\
\text { abnormal' }\end{array}$ & $\%$ age \\
\hline High & 128 & 11 & 8.6 \\
Medium & 169 & 15 & 8.9 \\
Low & 100 & 21 & $21 \cdot 0$ \\
\hline Total & 397 & 47 & \\
\hline
\end{tabular}

score roughly $1 \mathrm{SD}$ from the mean and is classified as 'doubtful'. A Woodside score of 4 relates to a Griffiths' score between 93 and 96, and a Woodside score of 5 relates to a Griffiths' score above 102. Of the 20 children assessed as 'doubtful' on the Woodside system, 15 were classified by the criterion test as 'abnormal' and five as 'normal'.

Of the 17 handicapped children included for comparative purposes, 13 had a Woodside total score (the combined scores of the four areas of the Woodside) of 0 , with corresponding Griffiths' mean general quotient score of less than 50 . The other four children had total scores of $11,7,3$, and 2, with the corresponding Griffiths general quotient scores being $71,78,63$, and 50 , respectively. The mean score for the 17 children on the Griffiths' general quotient was $42(\mathrm{SD}=20)$.

In Table 4 the numbers of children classified as 'normal', 'doubtful', and 'abnormal' by the Woodside screen are listed according to their socioeconomic state. In the low socioeconomic group 21 children were classified as 'abnormal' or 'doubtful' on the Woodside system. Statistically, this is nearly twice the number that would be expected by chance $\left(\mathrm{p}<0.025, \chi^{2}=8 \cdot 04\right)$.

The physical examination and the assessments of 'Hearing and vision' resulted in the referral of 14 physical defects ( $3 \%$ of 426$)$, 48 suspected hearing disabilities $(11 \%$ of 444$)$, and 40 suspected visual disabilities $(9 \%$ of 444$)$.

\section{Discussion and conclusions}

The Woodside system adapted for Australian conditions and used by a child health nurse was found to be a valid instrument with a Pearson's correlation coefficient of $r=0.70(p<0 \cdot 05)$ when compared with the widely accepted Griffiths' diagnostic test.

The highest correlations were in the areas of 'Gross motor' development $(\mathrm{r}=0 \cdot 8, \mathrm{p}<0 \cdot 001)$, and the lowest in the area of 'Social' development $(\mathrm{r}=0 \cdot 5, \mathrm{p}<0 \cdot 001)$. The latter findings could possibly be a consequence of the different environments of Glasgow and Adelaide. In Glasgow there is more limited indoor and outdoor living and recreation space as well as a poor climate, and it is suggested that there may be a greater pressure on the child to acquire acceptable social habits-for example, 'dry by day'-early.

Although $75 \%$ of 'suspected doubtfuls' were finally deemed to be 'abnormal', a close follow up of these children might be more appropriate than immediate referral. In this way developmental problems would not be missed, and parental anxieties associated with unnecessary over-referrals would be avoided. The cut off points between normal and doubtful and doubtful and abnormal may need reappraisal in the light of the recent observations by Hanson $e t$ al that the mean general quotient of the Griffiths' scales has increased. ${ }^{15}$ Nationwide representative figures from Hanson et al are awaited.

The percentage of referrals for hearing $(11 \%)$, vision $(9 \%)$, and physical $(3 \%)$ disabilities or defects are consistent with referral trends in two other Adelaide studies. ${ }^{14} 16$ These two studies were carried out on 4 year old children, and it is interesting to note that the referral rates for the various defects are similar to those in the present study of children aged $0-4$.

The only other developmental screening test that has been validated and encompasses the $0-4$ age range is the Denver developmental screening test. ${ }^{17}$ The impression is, however, that the Woodside system is less time consuming to apply (with eight test items compared with between 12-26 test items for the Denver screen), gives a better visual record of the progress of a child, and has a chart that is more suitable for use with the same child on more than one occasion.

Sheridan has defined a handicapped child as one 'who suffers from any continuing disability of body, intellect or personality which is likely to interfere with his normal growth and development or capacity to learn'. ${ }^{1}$ It is suggested that the Woodside system in the hands of trained child health nurses is a valuable instrument in helping to identify the present state of a child who may have a developmental handicap.

This study was supported by the Channel 10 Children's Medical Research Foundation Inc. Thanks are due to the parents for their enthusiastic cooperation, to Dr D Bowler for his consistent encouragement, and to Mr D Ness for his statistical analysis and advice.

\footnotetext{
References

1 Sheridan MD. Children's developmental progress. 5th ed. Windsor, UK: NFER Publishing, 1977.

${ }^{2}$ Bax M, MacKeith R. 'Does early recognition help?'. Dev Med Child Neurol 1969;11:411-2.
}

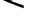
$\vec{\Phi}$ . 官 o

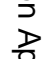
政 勇 
${ }^{3}$ Egan D, Illingworth RS, MacKeith RD. Developmental screening 0-5 years. Clinics in developmental medicine no 30. London: Heinemann, 1969.

${ }^{4}$ Loring J. The children who cannot wait. London: Spastic Society, 1971

${ }^{5}$ Starte GE. Developmental assessment of the young child in general practice. Practitioner 1974;213:823-8.

${ }^{6}$ Northern JL, Downs MP. Hearing in children. 2nd ed. Baltimore, USA: Williams and Wilkins, 1978.

${ }^{7}$ Norton-Taylor J. Vision-the critical years. Aust Fam Physician 1975:4:924-36.

${ }^{8}$ Gardiner P, MacKeith R, Smith V. Aspects of developmental medicine. no 32. London: Heinemann, 1969.

${ }^{9}$ Holt KS. Developmental paediatrics. Postgraduate paediatrics series. London: Butterworth and Co, 1977.

${ }^{10}$ Barber JH, Boothman R, Paget-Stanfield J. A new visual chart for pre-school developmental screening. Health Bull 1976; 34:80-91.

11 Stimson RS, Cleland EA. A socio-economic atlas of Adelaide. An analysis of the 1971 census. South Australia: Flinders University, 1975.
${ }^{12}$ Griffiths RG. The abilities of babies. High Wycombe, UK: The Test Agency, 1954.

${ }^{13}$ Griffiths RG. The abilities of young children. High Wycombe, UK: The Test Agency, 1970.

${ }^{14}$ Eu B. Health of the pre-school child. Med J Aust 1979;2:84-6.

15 Hanson R, Aldridge Smith J, Hume W. Achievements of infants on items of the Griffiths scales: 1980 compared with 1950. Child Care Health Dev 1985;2:91-104.

16 Johnstone O. Ill health, developmental delays in Adelaide 4 year old children. Aust Paediatr J 1980;16:248-54.

${ }^{17}$ Frankenburg WK, Goldstein AD, Camp BW. The revised Denver developmental screening test: its accuracy as a screening instrument. Pediatrics 1971;79:988-95.

Correspondence to Dr Bernice S L Eu, Special Services Unit, Child, Adolescent and Family Health Service, S A Health Commission, 285-295 South Terrace, Adelaide, South Australia 5000 .

Received 27 August 1985 